\title{
Calendar of Meetings
}

\author{
Donald R. Petersen \\ Greenleaf Associates \\ 6210 Siebert Street \\ Midland, MI 48640-27
}

\section{9-11 June 1999}

XI Symposium del Grupo Especializado de Cristalografia: Las interacciones responsables de la arquitectura del cristal. Alcalá de Henares (near Madrid), Spain. Preceded on June 8 by a workshop on the use of the Cambridge Structural Database. [Contact: Dra. Pilar Gómez-Sal, Universidad de Alcalá, Departamento de Química Inorgánica, Campus Universitario, 28871 Alcalá de Henares (Madrid), Spain. Tel: 34 (1) 885 4656; Fax: 34 (1) 885 4683; E-mail: pgomez@inorg.alcala.es; Info: http://www.inorg.alcala.es].

\section{June 1999}

Mineralogy of Ceramics, Ancient and Modern. London, England, UK. A meeting organized by the Applied Mineralogy Group of The Mineralogy Society, and held at The Mineralogy Society, 41 Queen's Gate, London SW7. [Contact: Andrew Middleton, Department of Scientific Research, British Museum, London WC1B 3DG, UK. Tel: 44 (171) 323 8342; E-mail: amiddleton@british-museum.ac.uk].

\section{7-19 June 1999}

Eighth Croation-Slovenian Crystallographic Meeting. Rovinj, Croatia. Held at the Hotel Istra, on Red Island, off Rovinj. The conference language, written and spoken, will be in English. [Contact: Prof. Boris Kamenar, Laboratory of General and Inorganic Chemistry, Faculty of Science, University of Zagreb, Ulica kralja Zvonimira 8, HR-10000 Zagreb, Croatia. Tel: 385 (1) 4606 673; Fax: 385 (1) 4611 191; E-mail: bkamenar@chem.pmf.hr; Info: http:// www.chem.pmf.hr/ hkz/cscm99].

\section{1-25 June 1999}

Nanostructures: Physics and Technology. St. Petersburg, Russia. [Contact: Boris Egorov, Ioffe Institute, Polytechnicheskaya st. 26, St. Petersburg 194 021, Russia. E-mail: boris.egorov@pop.ioffe.rssi.ru].

\section{June-1 July 1999}

$3^{\text {th }}$ Annual Meeting, Clay Minerals Society. West Lafayette, Indiana, USA. [Contact: Patricia Jo Eberl, Clay Minerals Society, Post Office Box 4416, Boulder, CO 80306, USA. Tel: 1 (303) 444 6405; Fax: 1 (303) 444 2260; E-mail: peber1@clays.org or Prof. Cliff Johnston, Department of Agronomy, 1150 Lilly Hall, Purdue University, West Lafayette, IN 47907-1150, USA. Tel: 1 (765) 496 1716; Fax: 1 (765) 496 2926; E-mail: clays@ @urdue.edu; Info: http://shadow.agry.purdue.edu/clay/claymin/cms 1999/ purdue 1999.html].

28-30 June 1999

ISPAC-12 $12^{\text {th }}$ International Symposium on Polymer Analysis and Characterization. La Rochelle, France. [Con- tact: Prof. Josef Janca, Universite de la Rochelle, Avenue Marillac, 17042 La Rochelle Cedex 01, France. Tel: 33 (546) 458 218; Fax: 33 (546) 458 243; E-mail: jjanca@cri.univIr.fr; Info: http://www.chem.cmu.edu/ispac/].

\section{June-2 July 1999}

Ninth International Symposium on Nondestructive Characterization of Materials. Darling Harbor (near Sydney), Australia. [Contact: Ms. Kim Howard, Center for Nondestructive Evaluation, 102 Maryland Hall, Johns Hopkins University, Baltimore, MD 21218, USA. Tel: 1 (410) 516 7126; Fax: 1 (410) 516 7249; E-mail: cnde@jhu.edu; Info: http://www.cnde.com].

\section{4-8 July 1999}

AISAS 99Australian International Symposium on Analytical Science. Melbourne, Australia. Held at the Melbourne Exhibition and Convention Centre. "Offers a scientific program of the highest quality, whilst providing an extensive trade exhibition and commercial workshops." [Contact: Prof. Philip Marriott, Department of Applied Chemistry, RMIT, GPO Box 2476V, Lonsdale Street, Melbourne, Vic. 3001, Australia. Tel: 61 (3) 9925 2632; Fax: 61 (3) 9639 1321; E-mail: aisas@ @mit.edu.au; Info: http:// www.rmit.edu.au/departments/cm/aisas99.html].

\section{4-9 July 1999}

Third International Conference on Borate Glasses, Crystals, and Melts: Structure and Applications. Sofia, Bulgaria. Held at the "Bistritsa Residence" (the Sofia International Management Center), located in the Bistritsa area of the foothills of Vitosha Mountain. The official language of the Conference is English. Topics specifically considered will include diffraction studies and structures of crystals, glasses, and melts. [Contact: Yanko B. Dimitriev, Department of Silicate Technology, University of Chemical Technology and Metallurgy, $8 \mathrm{~K} 1$. Ohridski Blvd., 1756 Sofia, Bulgaria. Tel: 359 (2) 681 120; Fax: 359 (2) 685 488; E-mail: yanko@adm1.uctm.acad.bg or A. C. Wright, J. J. Thomson Physical Laboratory, University of Reading, Whiteknights, Reading RG6 6AF, UK. Tel: 44 (118) 931 8555; Fax: 44 (118) 975 0203; E-mail: a.c.wright@ reading.ac.uk].

\section{5-9 July 1999}

ICCM-12 Twelfth International Conference on Composite Materials. Paris, France. Held in the Palais des Congrès de Paris. [Contact: ICCM-12 Conference Secretariat, MTE Bôite Postale 298, F-33605 Pessac, France. Tel: 33 (5) 5726 5342; Fax: 33 (5) 5726 5343; E-mail: orga@iccm12.org; Info: http://www.iccm12.org]. 


\section{5-30 July 1999}

International Conference on Analytical Chemistry: Analytical Science into the Next Millennium. Dublin, Ireland. [Contact: Malcolm R. Smyth, Dublin City University, Dublin 9, Ireland, Tel: 353 (1) 704 5308; Fax: 353 (1) 704 5032; E-mail: smythm@ccmail.dcu.ie].

\section{6-30 July 1999}

ICSOS-6 Sixth International Conference on Structure of Surfaces. Vancouver, British Columbia, Canada. Held at the University of British Columbia. [Contact: ICSOS-6 Secretariat, UBC Conference Center, 5961 Student Union Boulevard, Vancouver, B.C. V6T 2C9, Canada or Keith A. R. Mitchell, Department of Chemistry, University of British Columbia, Vancouver, B.C. V6T 1Z1, Canada. Tel: 1 (604) 822 5831; Fax: 1 (604) 822 2847; E-mail: karm@chem.ubc.ca; Info: http://www.conferences.ubc.ca/ conferences/events/icsos/icsos.htm].

\section{1-6 August 1999}

ACCGE-11 Eleventh American Conference on Crystal Growth and Epitaxy. Tucson, Arizona, USA. At Loews Ventana Canyon Resort. Related short course on Crystal Growth, July 31-August 1, same site. [Contact: Tony Gentile, ACCG-11 Secretariat, Post Office Box 3233, Thousand Oaks, CA 91359-0233, USA; Tel: 1 (805) 492 7047; Fax: 1 (805) 492 4062; E-mail: aacg@lafn.org; Info: http:// www.aml.arizona.edu/aacg/conferences/accge11/ accge 11.html]

\section{2-6 August 1999}

$\mathbf{4 8}^{\text {th }}$ Annual Denver X-ray Conference. Steamboat Springs, Colorado, USA. Held at the Sheraton Steamboat Resort. [Contact: Denise Flaherty, International Centre for Diffraction Data, 12 Campus Boulevard, Newtown Square, PA 19073-3273, USA. Tel:1 (610) 325 9814; Fax: 1 (610) 325 9823; E-mail: dxc@icdd.com; Info: http:// www.dxcicdd.com].

\section{4-13 August 199}

$18^{\text {th }}$ IUCr General Assembly and International Congress of Crystallography. Glasgow, Scotland, UK. Satellite meetings in connection with the Congress will include: Synchrotron Radiation (From Source to Science), Structural and Dynamical Aspects of Molecular and Ionic Solids Using Neutrons, Cambridge Crystallographic Databases and Software Systems, and the Crystallographic Computing School, held at various sites. The conference proper will be convened at the Scottish Exhibition and Conferences Centre in Glasgow, where several workshops will also be held on August 4. [Contact: Dr. C. J. Gilmore, Department of Chemistry, University of Glasgow, Glasgow G12 8QQ, UK. Tel: 44 (141) 339 8855; Fax: 44 (141) 330 4419; E-mail: iucr99@chem.gla.ac.uk or Prof. J. A. K. Howard, University of Durham, Department of Chemistry, South Road, Durham DH1 3LE, UK. Tel: 44 (191) 374 4647; Fax: 44 (191) 374 3745; E-mail: j.a.k.howard@durham.ac.uk; Info: http:// www.chem.gla.ac.uk/iucr99].
22-26 August 1999

$218^{\text {th }}$ American Chemical Society National Meeting. New Orleans, Louisiana, USA. Includes a three-day symposium on semicrystalline polymers, covering crystallization theory and simulations, crystallization kinetics, morphology, crystallization under flow, crystallization in copolymers and blends, and industrial/engineering aspects of semicrystalline polymers. [Contact: Prof. Hervé Marand, Department of Chemistry, Virginia Polytechnic Institute, Blacksburg, VA 24061-0212, USA. Tel: 1 (540) 231 8227; Fax: 1 (540) 231 8517; E-mail: hmarand@chemserver.chem.vt.edu; Info: http://membership.acs.org/p/pmse or http://www.acs.org/ meetings].

\section{2-27 August 1999}

18th International Conference on Amorphous and Microcrystalline Semiconductors. Snowbird, Utah, USA. [Contact: ICAMS18, University of Utah, Department of Physics, 115 S 1400 E, Room 201, Salt Lake City, UT 84112-0830, USA. E-mail: icams18@mail.physics.utah.edu; Info: http://icams18.physics.utah.edu].

\section{3-27 August 1999}

X-99 $18^{\text {th }}$ International Conference on X-ray and Innershell Processes. Chicago, Illinois, USA. [Contact: X-99 Conference Office, Physics Division/203-G122, Argonne National Laboratory, Argonne, IL 60439-4843, USA. Tel: 1 (630) 252 4044; Fax: 1 (630) 252 2864; E-mail: x99@anl.gov; Info: http://www.phy.anl.gov/x99].

\section{1-3 September 1999}

Faraday Discussion 114: Surface Science of Metal Oxides. Ambleside, England, UK. Held at University College of St. Martin. [Contact: Ms. S. Riaz, Royal Society of Chemistry, Burlington House, Piccadilly, London W1V OBN, UK. Tel: 44 (171) 437 8656; Fax: 44 (171) 434 1227; E-mail: riazs@rsc.org; Info: http://www.rsc.org/pdf/confs/ fara 114.pdf].

\section{1-4 September 1999}

ECNS'99 Second European Conference on Neutron Scattering. Budapest, Hungary. [Contact: Tamas Grûsz, Research Institute for Solid State Physics of the Hungarian Academy of Sciences, Neutron Physics Department, Post Office Box 49, H-1525 Budapest, Hungary. Tel: 36 (1) 395 9220; Fax: 36 (1) 395 9165; Info: http://www.kfki.hu/ ecns $99 /$ main.html].

\section{2-3 September 1999}

Micro and Surface Analysis by X-ray Beams. Ankara, Turkey. [Contact: Yavuz Ataman, Middle East Technical University, Department of Chemistry, Ankara 06531, Turkey. Tel: 90 (312) 210 3232; Fax: 90 (312) 210 1280; E-mail: xxxicsi@rorqual.cc.metu.edu.tr; Info: http://

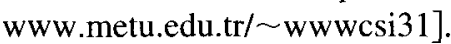

\section{4-8 September 1999}

Euroclay 1999 Conference of the European Clay Groups Association. Kraków, Poland. Held at the University of Mining and Metallurgy. A related two-day Workshop will be held following the meeting, in Banska Stiavnica, Slovakia. 
[Contact: Mrs. Aka Srodon, Euroclay 1999, Institute of Geological Sciences PAN, Senacka 1, 31-002 Kraków, Poland. Tel: 48 (12) 422 8920; Fax: 48 (12) 422 1609; E-mail: ndsrodon@cyr-kr.edu.pl; Info: http:/www.ingpan.krakow.pl/e_clay/eu_index.htm].

\section{2-17 September 1999}

Sixth International Conference on Surface X-ray and Neutron Scattering. Noordwijkerhout, The Netherlands. Held at the conference center Leewenhorst. Attendance is limited. [Contact: 6SXNS Secretariat, Philips Research Laboratories WB33, Prof. Holstlaan 4, 5656 AA Eindhoven, The Netherlands. Tel: 31 (40) 274 4551; Fax: 31 (40) 274 5002; E-mail: mfrankn@natlab.research.philips.com; Info: http:/www.research.philips.com/sxns/firs.html].

\section{3-17 September 1999}

PSD5 Fifth International Conference on PositionSensitive Detectors. London, England, UK. Held at University College London. [Contact: D. Bull, Centre for Advanced Instrumentation Systems, University College London, Gower Street, London WC1E 6BT, UK. Tel: 44 (171) 380 7838; Fax: 44 (171) 419 4318; E-mail: psd5@ucl.ac.uk; Info: http://www.hep.ucl.ac.uk/psd5].

\section{0-23 September 1999}

21st Durham Conference on X-ray Analysis. Durham, England, UK. [Contact: Sally Fuller, Philips Analytical, York Street, Cambridge CB1 2YA, UK. Tel: 44 (1223) 374411 ].

\section{0-24 September 1999}

ICQ7'99 Seventh International Conference on Quasicrystals. Stuttgart, Germany. At the University of Stuttgart (Vaihingen Campus). [Contact: ICQ7 Secretariat, Universität Stuttgart, Institut für Theoretische und Angewandte Physik, D-70550 Stuttgart, Germany. Tel: 49 (711) 685 5253; Fax: 49 (711) 685 5271; E-mail: icq7@itap.physik.unistuttgart.de; Info: http://icq7.uni-stuttgart.de].

\section{1-24 September 1999}

ECOSS-18 $18^{\text {th }}$ European Conference on Surface Science. Vienna, Austria. [Contact: ECOSS-18 Secretary, Institut für Allgemeine Physik, Technische Universität Wien, Wiedner Haupstrasse 8-10/134, A-1040 Vienna, Austria. E-mail: ecoss18-secretary@iap.tuwien.ac.at].

\section{7-29 September 1999}

ICDD: Pharmaceutical X-ray Powder Symposium. Newtown Square, Pennsylvania, USA. [Contact: Theresa Maguire, International Centre for Diffraction Data, 12 Campus Boulevard, Newtown Square, PA 19073-3273, USA. Tel: 1 (610) 325 9814; Fax: 1 (610) 325 9823; E-mail: maguire@icdd.com; Info: http://icdd.com].

\section{8-30 September 1999}

ECRS5 Fifth European Conference on Residual Stresses. Noordwijkerhout, The Netherlands. Held at the Conference Centre Leeuwenhorst, near The Hague. The conference language will be English. [Contact: Dr. Ir. Robert Delhez, Laboratory of Materials Science, Delft University of Technology, Rotterdamseweg 137, NL-2628 AL Delft, The Netherlands. Tel: 31 (15) 278 2261; Fax: 31 (15) 278 6730; E-mail: ecrs5@stm.tudelft.nl; Info: http://ecrs5.stm.tudelft.nl/ index.htm].

\section{September-2 October 1999}

Molecular Orientation in Polymers: Generation, Characterization, Application. Potsdam, Germany. [Contact: E-mail: epa99@canopus.physik.uni-potsdam.de; Info: http:// www.canopus.physik.uni-potsdam.de/ $\sim$ eps 99$]$.

\section{4-8 October 1999}

ECASIA'99 Eighth European Conference on Applications of Surface and Interface Analysis. Sevilla, Spain. [Contact: Jose Maria Sanz, Departimento de Fisica Aplicada, C-XII. Facultad de Ciencias, Universidad Autónoma de Madrid, Cantoblanco, E-28049 Madrid, Spain. Tel: 34 (1) 397 4508; Fax: 34 (1) 397 3669; E-mail: ecasia99@uam.es; Info: http://ecasia99.fa.uam.es].

\section{3-15 October 1999}

SRI'99 Eleventh National Conference on Synchrotron Radiation Instrumentation. Stanford, California, USA. [Contact: Suzanne Barrett, Conference Administrator, Stanford Synchrotron Radiation Laboratory, Mail Stop 99, Post Office Box 4349, Stanford, CA 94309-0201, USA. Tel: 1 (650) 926 3191; Fax: 1 (650) 926 3600; E-mail: barrett@ssrl.slac.stanford.edu; Info: http:// ssrl.slac.stanford.edu/sri99].

\section{3-16 October 1999}

Textures and Physical Properties of Rocks. Göttingen, Germany. [Contact: B. Leiss, IGDL-University of Göttingen, Goldschmidtstrasse 3, D-37077 Göttingen, Germany. E-mail: bleiss1@gwdg.de; Info: http://www.gwdg.de/ $\sim$ bleiss1/tppr.html].

\section{4-29 October 1999}

$26^{\text {th }}$ Annual Conference of the Federation of Analytical Chemistry and Spectroscopy Societies, in conjunction with the $\mathbf{4 6}^{\text {th }}$ International Conference on Analytical Sciences and Spectroscopy. Vancouver, British Columbia, Canada. [Contact: Prof. Michael Blades, Department of Chemistry, University of British Columbia, Vancouver, B.C. V6T 1Z1, Canada. Tel: 1 (604) 822 4468; E-mail: blades@chem.ubc.ca or FACSS, 1201 Don Diego Avenue, Santa Fe, NM 87505, USA. Tel: 1 (505) 820 1648; Fax: 1 (505) 989 1073; Info: http://facss.org/info.htm].

\section{4-19 November 1999}

Eastern Analytical Symposium. Somerset, New Jersey, USA. At the Garden State Convention Center. [Contact: Eastern Analytical Symposium, Post Office Box 633, Monchanin, DE 19710-0633, USA. Tel: 1 (302) 738 6218; Fax: 1 (302) 738 5275; Info: http://www.eas.org].

\section{November-3 December 1999}

Fall Meeting of the Materials Research Society. Boston, Massachusetts, USA. [Contact: Materials Research Society, 506 Keystone Drive, Warrendale, PA 15086-7573, USA. Tel: 1 (724) 779 3003; Fax: 1 (724) 779 8313; E-mail: info@mrs.org; Info: http://www.mrs.org]. 
March 2000

International Centre for Diffraction Data, Spring Meeting. Newtown Square, Pennsylvania, USA. Annual membership meeting. [Contact: Linda Shertz, International Centre for Diffraction Data, 12 Campus Boulevard, Newtown Square, PA 19073-3273, USA. Tel: 1 (610) 325 9814; Fax: 1 (610) 325 9823; E-mail: shertz@icdd.com; Info: http:// www.icdd.com].

\section{0-12 April 2000}

17th Biennial Conference, Australian Clay Mineral Society. Adelaide, South Australia, Australia. [Contact: Dr. Jock Churchman, CSIRO Land and Water, PMB\#2, Glen Osmond, S. A. 5064, Australia. Tel: 61 (8) 8303 8498; Fax: 61 (8) 8303 8550; E-mail: jock.churchman@adl.clw.csiro.au; Info: http://www.unisa.edu.au/acms/details.htm].

\section{1-13 April 2000}

MICRO 2000 Royal Microscopical Society Exhibition and Conference. London, England, UK. [Contact: Allison Winton, Royal Microscopical Society, $37 / 38$ St. Clements, Oxford OX4 1AJ, UK. Tel: 44 (1865) 248 768; Fax: 44 (1865) 791 237; E-mail: exhibitions@rms.org.uk; Info: http://www.rms.org.uk/mic2000.htm].

\section{4-28 April 2000}

Spring Meeting of the Materials Research Society. San Francisco, California, USA. [Contact: Materials Research Society, 506 Keystone Drive, Warrendale, PA 15086-7573, USA. Tel: 1 (724) 779 3003; Fax: 1 (724) 779 8313; E-mail: info@mrs.org; Info: http://www.mrs.org].

\section{0-23 May 2000}

EPDIC-7 Seventh European Powder Diffraction Conference. Barcelona, Spain. [Contact: Mrs. Gloria Casanova, UItramar Express, S. A., c/Diputació, 238-244, 3er, E-08007 Barcelona, Spain. Tel: 34 (93) 482 7140; Fax: 34 (93) 482 7158; E-mail: gcasanova@uex.es or epdic7@icmab.es; Info: http://www.icmab.es/epdic7].

\section{6-22 July 2000}

ICAM 2000 Sixth International Conference on Applied Mineralogy. Gottingen and Hanover, Germany. [Contact: ICAM 2000 Office, Post Office Box 510153, D-30631 Hanover, Germany. Tel; 49 (511) 643 2298; Fax: 49 (511) 643 3685; E-mail: icam2000@bgr.de; Info: http://www.bgr.de/ icam2000].

\section{1-26 July 2000}

ACA'00 American Crystallographic Association Annual Meeting. St. Paul, Minnesota, USA. [Contact: Info: http:// hwi.buffalo.edu/aca]

\section{July-4 August 2000}

$49^{\text {th }}$ Annual Denver X-ray Conference. Denver, Colorado, USA. Held at the Denver Marriott Tech Center Hotel. [Contact: Denise Flaherty, International Centre for Diffraction Data, 12 Campus Boulevard, Newtown Square, PA 190733273, USA. Tel: 1 (610) 325 9814; Fax: 1 (610) 325 9823; E-mail: dxc@icdd.com; Info: http://www.dxcicdd.com].
21-25 August 2000

SRI2000 $7^{\text {th }}$ International Conference on Synchrotron Radiation Instrumentation. Berlin, Germany. On the campus of Technische Universität Berlin. [Contact: E-mail: kongresse@wtb.tu-berlin.de: Info: http://sri2000.tu-berlin.de/ start.htm].

\section{3-8 September 2000}

Goldschmidt 2000: An International Conference for Geochemistry. Oxford, England, UK. [Contact: Goldschmidt 2000, Cambridge Publications, Post Office Box 27, Cambridge CBI 4GL, UK. Tel/Fax: 44 (1223) 333 428; E-mail: gold2000@campublic.co.uk; Info: http:// www.campublic.co.uk/science/conference/gold2000].

\section{2-30 September 2000}

$27^{\text {th }}$ Annual Conference of Federation of Analytical Chemistry and Spectroscopy Societies. Nashville, Tennessee, USA. [Contact: FACSS, 1201 Don Diego Avenue, Santa Fe, NM 87505, USA. Tel: 1 (505) 820 1648; Fax: 1 (505) 989 1073; Info: http://facss.org/info.htm].

\section{3-18 November 2000}

Eastern Analytical Symposium. Somerset, New Jersey, USA. At the Garden State Convention Center. [Contact: Eastern Analytical Symposium, Post Office Box 633, Monchanin, DE 19710-0633, USA. Tel: 1 (302) 738 6218; Fax: 1 (302) 738 5275; Info: http://www.eas.org].

\section{5-26 November 2000}

Annual Meeting, Crystallographic Society of Japan. Kyoto, Japan. [Contact: Info: http://wwwsoc.nacsis.ac.jp/ crsj/nenkai-e.html].

\section{November-1 December 2000}

Fall Meeting of the Materials Research Society. Boston, Massachusetts, USA. [Contact: Materials Research Society, 506 Keystone Drive, Warrendale, PA 15086-7573, USA. Tel: 1 (724) 779 3003; Fax: 1 (724) 779 8313; E-mail: info@mrs.org; Info: http://www.mrs.org].

\section{6-20 April 2001}

Spring Meeting of the Materials Research Society. San Francisco, California, USA. [Contact: Materials Research Society, 506 Keystone Drive, Warrendale, PA 15086-7573, USA. Tel: 1 (724) 779 3003; Fax: 1 (724) 779 8313; E-mail: info@mrs.org; Info: http://www.mrs.org].

July 2001

ACA'01 American Crystallographic Association Annual Meeting. Los Angeles, California, USA. [Contact: Info: http://hwi.buffalo.edu/aca].

\section{5-12 October 2001}

$28^{\text {th }}$ Annual Conference of Federation of Analytical Chemistry and Spectroscopy Societies. Detroit, Michigan, USA. [Contact: FACSS, 1201 Don Diego Avenue, Santa Fe, NM 87505, USA. Tel: 1 (505) 820 1648; Fax: 1 (505) 989 1073; Info: http://facss.org/info.htm]. 
26-30 November 2001

Fall Meeting of the Materials Research Society. Boston, Massachusetts, USA. [Contact: Materials Research Society,
506 Keystone Drive, Warrendale, PA 15086-7573, USA. Tel: 1 (724) 779 3003; Fax: 1 (724) 779 8313; E-mail: info@mrs.org; Info: http://www.mrs.org].

\section{Short Courses and Workshops}

\section{4-16 June 1999}

Rietveld Method Short Course. Atlanta, Georgia, USA. The Rietveld method is widely recognized to be uniquely valuable for structural analysis of nearly all crystalline materials not available as single crystals. This three-day intensive course will be conducted by Profs. R. B. Von Dreele, A. P. Wilkinson, and R. A. Young. The course will deal with the principles and techniques of Rietveld analysis and with avoiding or overcoming problems encountered in the use of it. The teaching system involves morning lectures, supervised individual hands-on experience running Rietveld refinements on PC computers in the afternoon, much studentteacher interaction and discussion, and availability of the computers at other times for individual practice. The student will have a choice of using either or both of the two widely used programs, DBWS and GSAS. Cost $\$ 995$, which includes a monograph and copies of computer programs and examples. [Contact: Continuing Education, Georgia Institute of Technology, Atlanta, GA 30332-0385, USA. Tel: 1 (404) 894 2547; E-mail: conted@gatech.edu; Info: http:// www.conted.gatech.edu].

\section{4-18 June 1999}

ICDD Clinic on X-ray Powder Diffraction: Advanced Methods. ICDD Headquarters, Newtown Square, Pennsylvania, USA. Covers computer-based methods of data collection and interpretation for both qualitative and quantitative phase analysis. Cost $\$ 1250$, includes textbook, printed lecture notes, use of computers where applicable, and lunch. [Contact: Manager, Schools and Conferences, International Centre for Diffraction Data, 12 Campus Boulevard, Newtown Square, PA 19073-3273, USA. Tel: 1 (610) 325 9814; Fax: 1 (610) 325 9823; E-mail: clinics@icdd.com; Info: http://www.icdd.com].

\section{5-17 June 1999}

Eighth Annual Fibre Diffraction and Non-crystalline Diffraction Workshop. St. Andrews, Scotland, UK. An activity of the Collaborative Computational Project for Fibre Diffraction, CCP13, devoted to developing and disseminating UNIX system software for fiber and polymer diffraction analysis. Includes a hands-on training session for new users of CCP13 and NCD software. [Contact: Alison Mutch, Daresbury Laboratory, Synchrotron Radiation Department, Warrington, Cheshire WA4 4AD, UK. Tel: 44 (1925) 603 363; Fax: 44 (1925) 603 124; E-mail: a.mutch@dl.ac.uk; Info: http:// www.dl.ac.uk/srs/ccp13/workshop99/workshop99.html].

\section{2-24 July 1999}

Eighth Annual ACA Summer Course in Crystallography. Athens, Georgia, USA. Held at the University of Georgia.
The first eight days will be devoted to basic crystallography, and will include lectures on the mathematics and physics behind structural analysis, the methods of structure solution, the refinement of atomic parameters, and the presentation and analysis of the results of a structure determination. The laboratory sessions will teach students to select and mount crystals, to determine unit cell dimensions, and to collect data on modern equipment. The final four days will cover macromolecular crystallography and include lectures on crystallization, data collection and processing, determination of heavy atom sites, structure solution by MIR, SIR, MAD, SAS and molecular replacement techniques, chain tracing, model building, and refinement methods. Instructors include co-directors Prof. Bi-Cheng Wang and Drs. Robert $\mathrm{A}$. Sparks, and Zhi-Jie Liu, John Rose, and Gary Newton. Tuition \$750; admission limited to 45-50 students. Accommodations available on campus. [Contact: Dr. John Rose, Department of Biochemistry and Molecular Biology, University of Georgia, Athens, GA 30602, USA. Tel: 1 (706) 542 1750; Fax: 1 (706) $542 \quad 3077$; E-mail: rose@bc14.biochem.uga.edu; Info: http://www.uga.edu/ $\sim$ biocryst/aca99.html].

\section{5-17 July 1999}

WAM1 IUPAC Workshop on Advanced Materials: Nanostructured Systems. Hong Kong, China. Held on the campus of Hong Kong University for Science and Technology. [Contact: Prof. M. A. El-Sayed, School of Chemistry and Biochemistry, Georgia Institute of Technology, Atlanta, GA 30332-0400, USA. Tel: 1 (404) 894 0292; Fax: 1 (404) 894 0294; E-mail: mostafa.el-sayed@chemistry.gatech.edu; Info: http://www.iupac.org/symposia/conferences/wam1/ index.html].

\section{July-1 August 1999}

Short Course on Crystal Growth. Tucson, Arizona, USA. Part of the Eleventh American Conference on Crystal Growth and Epitaxy, which follows at the same site. Held at Loews Ventana Canyon Resort. [Contact: Tony Gentile, ACCG-11 Secretariat, Post Office Box 3233, Thousand Oaks, CA 91359-0233, USA; Tel: 1 (805) 492 7047; Fax: 1 (805) 492 4062; E-mail: aacg@lafn.org; Info: http:// www.aml.arizona.edu/aacg].

\section{1-3 August 1999}

IUCr99 Satellite: Synchrotron Radiation, From Source to Science. Warrington, England, UK. Held at Daresbury Laboratory. The meeting will cover the use of synchrotron radiation in crystallographic and structural studies. The meeting fee is $£ 150$, which includes all meals and transport to Glasgow on August 4. Accommodations, available locally, are 